\title{
Continuously advancing quality care
}

Aoife Molloy, Clinical Lead for the NHS England Evidence-Based Interventions programme

Terence Stephenson \& Martin Marshall, Co-Chairs of the independent Expert Advisory Committee

Paediatricians have a role in advocating that children should not receive treatments which don't work which then frees up time for treatments which do work.

The Evidence-Based Interventions programme is a clinically led programme which uses the latest evidence to reduce inappropriate care by publishing guidance for tests, treatments and procedures proven to be inappropriate in some circumstances or which can sometimes do more harm than good [1]. It is a joint enterprise between national partners: The Academy of Medical Royal Colleges (AoMRC), NHS Clinical Commissioners (NHSCC), the National Institute for Health and Care Excellence (NICE) as well as NHS England and NHS Improvement.

The program's independent Expert Advisory Committee was invited to develop an approach to select interventions and agree guidance. The Committee compiled a list of interventions with no or limited clinical effectiveness, based on clinical evidence and research including NICE guidelines, Choosing Wisely recommendations and peer reviewed publications [2]. It particularly looked at surgical interventions and diagnostics where there was high variability in the application of clinical guidelines. It also worked with the relevant specialist clinical societies and patients to refine the list.

This is not about rationing. The aim of the programme is to reduce avoidable harm to patients; to save precious professional time; to maximise value and avoid waste; to help clinicians keep up to date with the changing evidence base and best practice; to drive innovation and to accelerate the adoption of new and proven innovations by reducing the number of inappropriate interventions; and to reduce unwarranted variation. The data show that there are wide disparities between the age-sex standardised rate per 100,000 population of procedures carried out in different regions of the country. In many cases there is not rational explanation for this other than differences in medical practice and variability in implementation of best available evidence and guidelines.

Here we use one the 31 interventions highlighted in the 2020 phase of the program which relates to children to illustrate the principles at work.

NICE guidance [3] recommends that adjuvant adenoidectomy should not be performed for the treatment of glue ear in the absence of persistent and/or frequent upper respiratory tract symptoms. The benefit in hearing compared to grommets alone is very limited. Risks of adenoidectomy include damage to teeth, lips or gums, bleeding; risks associated with sedation/anaesthetic; and perhaps longer term disbenefits [4]. Evidence underpinning NICE guidance is under regular surveillance to check if updates need to be made to NICE guidance. The Evidence Based Interventions guidance will be updated as appropriate [5]. 
One of the most striking concerns we have had over the last decade is the huge variation in practice across England as highlighted by the publication of the Atlas of Variation. There were 1,921 operations to remove adenoids during 2018/19 but there was a greater than 13fold variation across England. The population of England is divided into over one hundred Clinical Commissioning Groups (CCG's), each covering from 100,000 to $1.8 \mathrm{~m}$ people. The figure shows that the rate of adenoidectomy varies across these CCG's from 1 to 13 per 100,000 age-sex standardised population under 19 years of age.

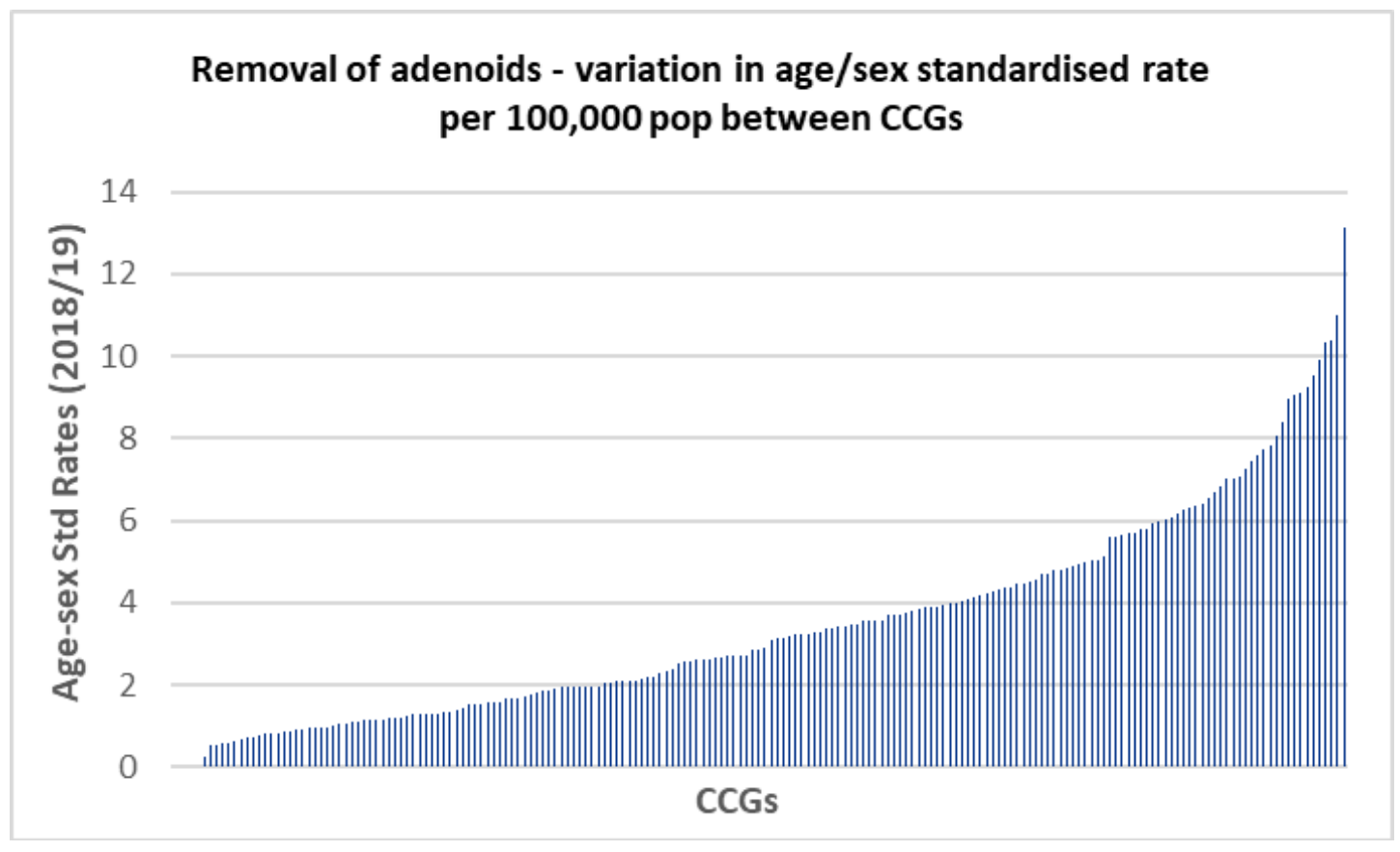

Adenoidectomy can still be carried out in children if clinically justified but the key is that it should not be a routine accompaniment to insertion of grommets for chronic conductive hearing loss. By only offering this operation routinely on the NHS in specific, appropriate cases, the aim is to reduce the rate of this procedure to the $25^{\text {th }}$ centile for the country, which would free up 1,131 operating slots per annum for operations which children do benefit from.

This recommendation has been agreed with the British Association for Paediatric Otolaryngology, British Association of Otorhinolaryngology (ENT UK) and the Royal College of Paediatrics \& Child Health.

This evidence-based approach to restricting inappropriate interventions offered to children and families is an initiative which should be adopted globally to optimise the use of scarce resources, especially given the pressure on health services following the COVID-19 pandemic. The need for such initiatives will never cease - medicine constantly evolves and we need to continually review stopping doing things which modern evidence shows don't work rather than continuing to practise in the way which we have always done. However, this requires us all to challenge deeply embedded beliefs and practices and we should not underestimate how difficult culture change like this is in practice. 


\section{References:}

1] Expert Advisory Committee to the NHS England Evidence-Based Intervention Programme: Evidence-Based Interventions List 2: Guidance - first published November 2020 https://www.aomrc.org.uk/ebi/wave-two-guidance/

2] https://blogs.bmj.com/bmjleader/2020/07/20/leading-wisely-in-the-post-covid-19-eraby-dr-ole-tjomsland-and-dr-jan-frich/

3] NICE Guidance. https://www.nice.org.uk/Guidance/CG60

4] Byars SG, Stearns SC, Boomsma JJ. Association of Long-Term Risk of Respiratory, Allergic, and Infectious Diseases with Removal of Adenoids and Tonsils in Childhood. JAMA Otolaryngol Head Neck Surg. 2018;144(7):594-603.

doi:10.1001/jamaoto.2018.0614

5] https://www.nice.org.uk/guidance/cg60/resources/2018-surveillance-of-otitis-mediawith-effusion-in-under-12s-surgery-nice-guideline-cg60-6604581853/chapter/Surveillancedecision?tab=evidence

\section{Acknowledgements:}

The authors would like to acknowledge ENTUK for their support in the development and implementation of the Evidence Based Interventions guidance for adenoidectomy, in particular Professor Nirmal Kumar, Mr Andrew Swift, Mr Andrew Marshall and Mr Arun Takhar. 